\title{
NEW EXISTENCE THEOREMS FOR NONLINEAR EQUATIONS OF HAMMERSTEIN TYPE
}

\author{
BY \\ W. V. PETRYSHYN( $\left.{ }^{(}\right)$AND P. M. FITZPATRICK( $\left.{ }^{2}\right)$
}

\begin{abstract}
Let $X$ be a real Banach space, $X^{*}$ its dual, $A$ a linear map of $X$ into $X^{*}$ and $N$ a nonlinear map of $X^{*}$ into $X$. Using the recent results of Browder and Gupta, Brezis, and Petryshyn, in this paper we study the abstract Hammerstein equation, $w+A N w=0$. Assuming suitable growth conditions on $N$, new existence results are obtained under the following conditions on $X, A$ and $N$. In $\S 1: X$ is reflexive, $A$ bounded with $f(x)=(A x, x)$ weakly lower semicontinuous, $N$ bounded and of type (M). In $\S 2: X$ is a general space, $A$ angle-bounded, $N$ pseudo-monotone. In $\S 3: X$ is weakly complete, $A$ strictly (strongly) monotone, $N$ bounded (unbounded) and of type (M). In $\$ 4: X$ is a general space, $A$ is monotone and symmetric, $N$ is potential. In $\S 5: X$ is reflexive and with Schauder basis, $X^{*}$ strictly convex, $N$ quasibounded and either monotone, or bounded and pseudo-monotone, or bounded and of type (M).
\end{abstract}

Introduction. Let $X$ be a real Banach space, $X^{*}$ its conjugate space and $(w, x)$ the value of the functional $w$ in $X^{*}$ at the element $x$ in $X$. In this paper we study the solvability of functional equations of the form

$$
w+A N w=0 \quad\left(w \in X^{*}\right)
$$

under various conditions on $X$, on the linear mapping $A$ of $X$ into $X^{*}$ and the nonlinear mapping $N$ of $X^{*}$ into $X$. The class of equations (1) includes nonlinear integral equations of Hammerstein type

$$
w(x)+\int_{D} K(x, y) f(y, w(y)) d y=0,
$$

where $D$ is a measure space with $\sigma$-finite measure $d y$, the unknown function $w(x)$ is defined on $D$ and the linear and nonlinear mappings $A$ and $N$ are given by

$$
A v(x)=\int_{D} K(x, y) v(y) d y, \quad N w(x)=f(x, w(x)) .
$$

Received by the editors April 27, 1970 and, in revised form, September 23, 1970.

AMS 1969 subject classifications. Primary 4780; Secondary 4530, 4610, 4690.

Key words and phrases. Existence theorems, nonlinear operators, Hammerstein equations, weakly lower semicontinuous functionals, monotone and pseudo-monotone operators, operators of type (M), pseudo- $A$-proper operators, angle-bounded operators, potential operators, weakly and hemicontinuous operators.

(1) Supported in part by NSF grant GP-8556.

${ }^{(2)}$ Supported by NDEA Fellowship, Title IV.

Copyright (C) 1971, American Mathematical Society 
The study of equation (2) with $A$ and $N$ given by (3) has been initiated by Hammerstein [12] and continued by Inglisch [13], Nemyckir [18], Golomb [10] and others. Further studies have been carried out by Rothe [21], Dolph [7], Vaĭnberg [22], Krasnosel'skir [17], and others (see [20], [22]). In recent years the variational and the monotonicity methods are most often used in the study of the solvability of equations (1) and (2).

Vainnberg [22] and others applied variational methods to the problem of solving (2). By imposing suitable conditions on $A$ and $N$, they transformed the problem of solving (2) in $L^{p}(D)$ into the problem of solving a suitable equation

$$
T x=0 \quad\left(x \in L^{2}(D)\right)
$$

in the Hilbert space $L^{2}(D)$ with $T$ a potential operator in $L^{2}(D)$ such that its potential had a local minimum which then was used as a solution of equation (4).

The monotonicity method for equation (1) with operators $A$ and $N$ acting in a Hilbert space has been successfully used by Vainnberg [22], [23], Kolodner [14], Kolomy [15], [16], and especially by Dolph and Minty [8]. More recent papers applying the monotone operator theory to abstract Hammerstein equations (1) in Banach spaces include Amann [1], [2], [3], Brezis [6], Vaĭnberg [24], DeFigueiredo-Gupta [9], Browder-Gupta [4], VaǏnberg-Lavrent'ev [25], BrowderDeFigueiredo-Gupta [5], and others.

The above authors studied equations (1) and/or (2) under the basic assumption that $N$ is either potential or monotone. In generalizing the Dolph-Minty [8] result to reflexive strictly convex Banach spaces $X$ and $X^{*}$, Brezis [6] established an existence theorem for (1) for a more general class of mappings $N$, namely, those of type (M). The definition of a mapping of type (M) given by Brezis is, as we shall discuss later, somewhat different than that given in this paper.

The purpose of this paper is to carry further the study of the solvability of the abstract Hammerstein equation (1) for $N$ not necessarily monotone and bounded and/or for nonseparable $X$ and $X^{*}$, which are not necessarily reflexive and strictly convex. In this paper we obtain new existence results which we now outline briefly.

In $\S 1$ we study (1) under the assumption that $X$ is reflexive, $A: X \rightarrow X^{*}$ is linear and bounded, and $N: X^{*} \rightarrow X$ bounded and of type (M). It is first shown (Theorem 1) that if $f(x)=(A x, x), \forall x \in X$, is weakly lower semicontinuous and

$$
\left(A^{*} x, x\right)+\left(N A^{*} x, A^{*} x\right) \geqq 0 \text { for all } x \in \dot{B}(0, r) \text { and some } r>0,
$$

then (1) is solvable; special cases are considered. If we strengthen the condition on $A$ by assuming that $A$ is angle-bounded with constant of angle-boundedness $c$, and $N$ satisfies either the growth condition (Theorem 2)

$$
(N u, u) \geqq-k\|u\|^{2}+(N(0), u) \quad \forall u \in X^{*} \text { and some } k \geqq 0
$$

with $k\left(1+c^{2}\right)\|A\|<1$ or the growth condition (Theorem 3)

$$
N x\|\leqq \gamma\| x \| \quad \forall x \in X \quad \text { such that }\|x\|>r \text { and some } \gamma>0
$$

with $\gamma\left(1+c^{2}\right)\|A\|<1$, then (1) is solvable. 
In $\S 2$ we study (1) for $X$ a general Banach space, $A$ angle-bounded and $N$ pseudomonotone. The solvability of (1) is proved for $N$ satisfying either the growth condition (6) (Theorem 4) or the growth condition (7) (Theorem 5).

In $\S 3$ we show (Theorems 6 and 7) that the reflexivity condition on $X$ in Theorems 2 and 3 can be relaxed to that of weak completeness of $X$ if the monotonicity condition on $A$ in the angle-boundedness requirement is strengthened to strict monotonicity. If we strengthen further the condition on $A$ by assuming that $A$ is strongly monotone, then Theorems 2 and 3 are valid for mappings $N$ of type (M) for which the boundedness condition is somewhat relaxed (Theorems 8 and 9).

In $\$ 4$ a variational method is first used (Theorems 10 and 11) to extend certain Hilbert space results of Vaǐnberg to general Banach spaces under the assumption that $A: X \rightarrow X^{*}$ is a monotone symmetric mapping while $N: X^{*} \rightarrow X$ is a potential mapping satisfying suitable growth conditions. Our Theorem 12 illustrates the naturalness and usefulness of growth condition (6).

In $\S 5$ we strengthen certain results of $\S 1$ in case $X$ is a reflexive space with a Schauder basis, $X^{*}$ is strictly convex and $A N: X^{*} \rightarrow X^{*}$ is quasibounded and either monotone, bounded and pseudo-monotone, or bounded and of type (M).

Finally, we remark that for our purposes the result of Browder-Gupta [4] concerning the existence of a suitable Hilbert space in which to split the operator $A$, the extension of some of the results of Brezis [6], and the results of Petryshyn [19] concerning the solvability of equations involving pseudo-monotone mappings and mappings of type (M) under various "at infinity" conditions will be of special usefulness.

1. In this section we study (1) under the assumption that $X$ is reflexive and the nonlinear mapping $N$ is of type (M).

Definition 1. Let $X$ be a Banach space and $T: X \rightarrow X^{*}$. Then $T$ is said to be of type (M) provided the following two conditions hold:

(i) If $x_{n} \rightarrow x$ in $X, T\left(x_{n}\right) \rightarrow g$ in $X^{*}$ and $\lim \sup _{n}\left(T\left(x_{n}\right), x_{n}\right) \leqq(g, x)$, then $T(x)=g$,

(ii) $T$ is continuous from finite-dimensional subspaces of $X$ into $X^{*}$ equipped with the weak* topology.

The definition of a mapping of type (M) given above is different than that given by Brezis [6]. In [6] a mapping of type (M) need not act from $X$ to $X^{*}$, but may act from $X$ to $Y$, where there is a bilinear form on $X \times Y$. In addition, if one replaces sequences by filters and weak topology by the topology induced by the bilinear form in the definition above, then one obtains Brezis' definition. When $Y=X^{*}$ it is clear that a mapping satisfying Brezis' definition satisfies the one given here. However, as remarked by Brezis, in order to guarantee that the two definitions coincide, $X$ must be reflexive and separable $\left({ }^{3}\right)$.

$\left({ }^{3}\right)$ We thank the referee for suggesting that we comment upon the difference between Brezis' definition of type (M) and the one given here. 
It was shown in [6] that monotone hemicontinuous operators as well as pseudomonotone operators (to be defined later) from $X$ to $X^{*}$ are both of type (M). The sum of two operators of type (M) need not be of type (M). However, we do have the following result.

Proposition 1.1. Let $X$ be a Banach space and suppose $T: X \rightarrow X^{*}$ is of type (M). Suppose that $S: X \rightarrow X^{*}$ is weakly continuous (i.e. $x_{n} \rightarrow x \Rightarrow S\left(x_{n}\right) \rightarrow S(x)$ ) and that the functional $f(x)=(S(x), x)$ is weakly lower semicontinuous (i.e., $\left.x_{n} \rightarrow x \Rightarrow f(x) \leqq \lim \inf _{n} f\left(x_{n}\right)\right)$. Then the mapping $T+S: X \rightarrow X^{*}$ is also of type (M).

Proof. Let $\left\{x_{n}\right\} \subset X$ be such that $x_{n} \rightarrow x,(T+S)\left(x_{n}\right) \rightarrow g$ and

$$
\lim _{n} \sup \left((T+S)\left(x_{n}\right), x_{n}\right) \leqq(g, x) .
$$

Since $S$ is weakly continuous it follows that $S\left(x_{n}\right) \rightarrow S(x)$ and hence $T\left(x_{n}\right) \rightarrow g-S(x)$. Now

$$
\begin{aligned}
\limsup _{n}\left(T\left(x_{n}\right), x_{n}\right) & =\lim _{n} \sup \left\{\left((T+S)\left(x_{n}\right), x_{n}\right)-\left(S\left(x_{n}\right), x_{n}\right)\right\} \\
& \leqq \lim _{n} \sup \left((T+S)\left(x_{n}\right), x_{n}\right)-\lim _{n} \inf \left(S\left(x_{n}\right), x_{n}\right) \\
& \leqq(g-S(x), x) .
\end{aligned}
$$

Since $T$ is of type (M) it follows that $T(x)=g-S(x)$, and consequently $(T+S)(x)$ $=g$. Thus $T+S$ is of type (M). Q.E.D.

REMARK 1.1. If $T: X \rightarrow X^{*}$ is monotone (i.e., $(T(x)-T(y), x-y) \geqq 0$ for all $x, y \in X)$ and weakly continuous, then the functional $f: X \rightarrow R$ defined by $f(x)=(T(x), x)$ for all $x \in X$ is weakly lower semicontinuous. Indeed, suppose $x_{n} \rightarrow x$. Then since $\left(T\left(x_{n}\right)-T(x), x_{n}-x\right) \geqq 0$ we see that $\left(T\left(x_{n}\right), x_{n}-x\right)$ $\geqq\left(T(x), x_{n}-x\right)$. Hence

$$
\lim _{n} \inf \left(T\left(x_{n}\right), x_{n}-x\right) \geqq \lim _{n} \inf \left(T(x), x_{n}-x\right)=0 .
$$

Since

$$
\lim _{n} \inf \left(T\left(x_{n}\right), x_{n}-x\right)=\lim \inf \left(T\left(x_{n}\right), x_{n}\right)-(T(x), x),
$$

it follows that $\lim \inf _{n} f\left(x_{n}\right) \geqq f(x)$. Thus $f$ is weakly lower semicontinuous.

REMARK 1.2. If $T: X \rightarrow X^{*}$ is completely continuous (i.e., if $\left\{x_{n}\right\} \subset X$ is such that $x_{n} \rightarrow x$, then $\left.T\left(x_{n}\right) \rightarrow T(x)\right)$, then clearly the functional $g: X \rightarrow R$ defined by $g(x)=(T(x), x)$ is weakly lower semicontinuous.

From Remarks 1.1 and 1.2 one sees that if $T: X \rightarrow X^{*}$ is of type (M), $S: X \rightarrow X^{*}$ is weakly continuous and monotone and $L: X \rightarrow X^{*}$ is completely continuous, then $T+S+L$ is of type (M). In [6] Brezis observed that $T+S$ was of type (M). It is not the case, as is shown by the following example, that the sum of an operator of type (M) and a compact operator (i.e., continuous and the images of bounded sets are precompact) is always of type (M). 
EXAMPLE. Let $X=l_{2}$. Since the zero map is clearly of type (M) it suffices to find a map $T$ of $l_{2}$ into $l_{2}$ such that it is compact and not of type (M). For $x \in l_{2}$ define $T(x)=\langle 1-\|x\|, 0,0, \ldots\rangle$. Then $T$ is compact. Let $x^{n}=\left\langle x_{1}^{n}, x_{2}^{n}, \ldots\right\rangle$ where $x_{j}^{n}=\frac{1}{2} \delta_{n j}$, with $\delta_{n j}=1$ if $n=j$ and $\delta_{n j}=0$ for $n \neq j$. Since $T\left(x^{n}\right)=\left\langle\frac{1}{2}, 0,0, \ldots\right\rangle$ for all $n$, we see that $x^{n} \rightarrow 0, T\left(x^{n}\right) \rightarrow\left\langle\frac{1}{2}, 0,0, \ldots\right\rangle=g$, and $\left.\lim \sup _{n}\left\{T\left(x_{n}\right), x_{n}\right)\right\}$ $=0 \leqq(g, 0)$. Since $T(0) \neq g$ we see that $T$ is not of type (M). Q.E.D.

In what follows we shall utilize the following two results for maps of type (M) which will prove to be essential in our study of Hammerstein equations. Our arguments are similar to those used by Browder, Brezis, DeFigueiredo, and others in the study of monotone type mappings.

Proposition 1.2. Let $X$ be a reflexive Banach space and $T: X \rightarrow X^{*}$ a mapping of type (M) which satisfies the following condition:

( $\beta)$ If $\left\{x_{j}\right\}$ is a bounded sequence in $X$ such that $\left\{\left(T x_{j}, x_{j}\right)\right\}$ is also bounded, then $\left\{T\left(x_{j}\right)\right\}$ is bounded.

If there exists an $r>0$ such that $(T(x), x) \geqq 0$ for $x \in \dot{B}(0, r)$, then there exists an $x_{0} \in \bar{B}(0, r)$ such that $T\left(x_{0}\right)=0$.

Proof. Let $\mathscr{F}$ be the family of all finite-dimensional subspaces of $X$ ordered (partially) by inclusion. For each $F$ in $\mathscr{F}$, let $J_{F}$ be the inclusion map of $F$ into $X$, $J_{F}^{*}$ the dual projection map of $X^{*}$ onto $F^{*}$, and $T_{F}=J_{F}^{*} T J_{F}$ the continuous map of $F$ into $F^{*}$. Since, for each $x$ in $\dot{B} \cap F,\left(T_{F}(x), x\right)=(T(x), x) \geqq 0$, it follows that there exists an $x_{F}$ in $\bar{B} \cap F$ such that $T_{F}\left(x_{F}\right)=0$. For each $F \in \mathscr{F}$, let $A_{F}=\left\{x \in F \mid T_{F}(x)=0\right.$, $\|x\| \leqq r\}$, let $V_{F}=\bigcup_{F \in \mathscr{F}, F^{1} \supset F}\left\{A_{F^{1}}\right\}$, and let $\tilde{V}_{F}=$ weak cl $\left(V_{F}\right)$. Then for each $F \in \mathscr{F}$, $\widetilde{V}_{F}$ is weakly compact, and the family $\left\{\tilde{V}_{F} \mid F \in \mathscr{F}\right\}$ obviously has the finite intersection property. Hence $\bigcap_{F \in \mathscr{F}} \tilde{V}_{F} \neq \varnothing$ and therefore there exists in $\bar{B}(0, r)$ an element $x_{0}$ which lies in $\tilde{V}_{F}$ for each $F$ in $\mathscr{F}$. We claim that $T\left(x_{0}\right)=0$. Indeed, if $T\left(x_{0}\right)$ were not 0 , then there would exist an $u_{0}$ in $X$ such that

$$
\left(T\left(x_{0}\right), u_{0}\right)<0 .
$$

Now, let $F$ be an element of $\mathscr{F}$ which contains the points $x_{0}$ and $u_{0}$. Now since $x_{0}$ lies in $\tilde{V}_{F}$, there exists a sequence $\left\{x_{F}\right\} \equiv\left\{x_{j}\right\}$ in $V_{F}$ such that $x_{j} \rightarrow x_{0}$ in $X$. Each $x_{j}$ lies in a finite-dimensional subspace $F_{j}$ of $X$ which contains $F$ and $x_{j}$ satisfies the equation $T_{j}\left(x_{j}\right)=0$. Since $x_{j} \rightarrow x_{0}$ in $X$ and $0=\left(T_{j}\left(x_{j}\right), x_{j}\right)=\left(T x_{j}, x_{j}\right)$ for each $j$, condition $(\beta)$ implies the boundedness of $\left\{T\left(x_{j}\right)\right\}$. In view of this and the reflexivity of $X$, we may assume that $T\left(x_{j}\right) \rightarrow g$ for some $g$ in $X^{*}$. Now, since both $x_{j}$ and $x_{0}$ lie in $F_{j}$ for each $j,\left(T\left(x_{j}\right), x_{j}-x_{0}\right)=\left(T_{j}\left(x_{j}\right), x_{j}-x_{0}\right)=0$; therefore,

$$
\begin{aligned}
\lim \sup \left(T\left(x_{j}\right), x_{j}\right) & =\lim \sup \left(T\left(x_{j}\right), x_{0}\right) \\
& =\lim \left(T\left(x_{j}\right), x_{0}\right)=\left(g, x_{0}\right) .
\end{aligned}
$$

Hence $x_{j} \rightarrow x_{0}$ in $X, T\left(x_{j}\right) \rightarrow g$ in $X^{*}$, and lim sup $\left(T\left(x_{j}\right), x_{j}\right)=\left(g, x_{0}\right)$. Consequently, since $T$ is of type (M), $T\left(x_{0}\right)=g$. Now, $\left(T\left(x_{j}\right), u_{0}\right)=0$ for each $j$, and therefore $\lim _{j}\left(T\left(x_{j}\right), u_{0}\right)=\left(g, u_{0}\right)=0$. Since $T\left(x_{0}\right)=g$, we have $\left(T\left(x_{0}\right), u_{0}\right)=0$, in contradiction to $(l)$. It follows that $T\left(x_{0}\right)=0$. Q.E.D. 
We add that condition $(\beta)$ is analogous to the requirement that $T$ be strongly quasibounded as defined by Browder and Hess in their paper, Nonlinear mappings of monotone type in Banach spaces, a preliminary version of which the authors obtained from D. G. DeFigueiredo.

Proposition 1.3. Let $X$ be a reflexive Banach space with $\operatorname{dim}(X) \geqq 2$ and $T: X \rightarrow X^{*}$ a map of type (M) which satisfies condition $(\beta)$ of Proposition 1.2. Suppose there exists an $r>0$ such that for all $x$ with $\|x\| \geqq r,(T(x), x) \neq 0$, then there exists an $x_{0}$ in $X$ such that $T\left(x_{0}\right)=0$.

Proof. Let $\mathscr{F}^{\prime}$ be the family of all finite-dimensional subspaces of $X$ of dimension greater than 1. Using the same notation as in Proposition 1.2 it is clear that if we can show that $T_{F}(x)=0$ has a solution in $\bar{B}(0,2 r) \cap F$ for each $F \in \mathscr{F}^{\prime}$, then the same proof as that for Proposition 1.2 will suffice. Indeed, let $F \in \mathscr{F}^{\prime}$. Then $T_{F}: F \rightarrow F^{*}$ is continuous and hence the functional $f: F \rightarrow R$, defined by $f(x)$ $=\left(T_{F}(x), x\right)$, is continuous. Since $F-\bar{B}(0, r)$ is connected, $T_{F}(F-\bar{B}(0, r))$ is connected, and since $0 \notin T_{F}(F-\bar{B}(0, r))$ we see that either $\left(T_{F}(x), x\right)>0$ for all $x \in F$ with $\|x\|>r$, or $\left(T_{F}(x), x\right)<0$ for all $x \in F$ with $\|x\|>r$. In the first case clearly $T_{F}(x)=0$ has a solution in $\bar{B}(0,2 r)$, and in the second case we consider $-T_{F}$, which satisfies $\left(-T_{F}(x), x\right)>0 \forall x \in F$ with $\|x\|=2 r$, and hence $-T_{F}(x)=0$ has a solution, and hence $T_{F}(x)=0$ has a solution. Q.E.D.

We note that when $X$ is separable and reflexive, and condition $(\beta)$ is replaced by boundedness, then Propositions 1.2 and 1.3 follow from the results of Brezis [6].

We add that our arguments in the proof of Proposition 1.2 are similar to those used by DeFigueiredo (in a paper to be published) who extended the surjectivity theorem of Brezis to nonseparable reflexive Banach spaces by showing that if $X$ is reflexive and $T: X \rightarrow X^{*}$ is a bounded coercive map of type (M), then $T$ is onto. Since the boundedness of $T$ implies condition $(\beta)$ the surjectivity result of DeFigueiredo follows as a corollary of Proposition 1.2.

In this section we obtain two existence theorems for the abstract Hammerstein equation

$$
w+A N(w)=0 \quad\left(w \in X^{*}\right)
$$

for the case when $X$ is a reflexive Banach space, $N: X^{*} \rightarrow X$ is a bounded mapping of type (M) and $A: X \rightarrow X^{*}$ is a suitable linear mapping.

Since $X$ is reflexive, to solve equation (1.1) it suffices to solve the equation

$$
A^{*}(x)+A N A^{*}(x)=0 \quad(x \in X),
$$

where $A^{*}$ is the adjoint mapping of $X^{* *}=X$ into $X^{*}$.

To prove our first theorem we will need the following result.

Proposition 1.4. Let $X$ be a reflexive Banach space. Suppose $A: X \rightarrow X^{*}$ is a bounded linear mapping such that the functional $f: X \rightarrow R$ defined by $f(x)=(A(x), x)$, 
$x \in X$, is weakly lower semicontinuous. If $N: X^{*} \rightarrow X$ is of type (M) and bounded, then the mapping

$$
T=A^{*}+A N A^{*}: X \rightarrow X^{*}
$$

is also of type (M) and bounded.

Proof. Since $A^{*}: X \rightarrow X^{*}$ is weakly continuous and the functional $g: X \rightarrow R$ defined by $g(x)=\left(A^{*}(x), x\right)=(A(x), x)$ is weakly lower semicontinuous, we see, by Proposition 1.1, that to prove that $A^{*}+A N A^{*}$ is of type (M), it suffices to show that $A N A^{*}$ is of type (M).

Suppose $\left\{x_{n}\right\} \subset X$ is such that $x_{n} \rightarrow x, A N A^{*}\left(x_{n}\right) \rightarrow g$ and $\lim \sup _{n}\left(A N A^{*}\left(x_{n}\right), x_{n}\right)$ $\leqq(g, x)$. Since $A^{*}$ is linear and bounded, $A^{*}\left(x_{n}\right) \rightarrow A^{*}(x)$, and hence, since $N$ is bounded, $\left\{N A^{*}\left(x_{n}\right)\right\}$ is bounded. But $X$ is reflexive, and hence we may choose a subsequence $\left\{x_{n_{k}}\right\}$ such that $N A^{*}\left(x_{n_{k}}\right) \rightarrow f$. Thus $A N A^{*}\left(x_{n_{k}}\right) \rightarrow A(f)$, and consequently $A(f)=g$. Now

$$
\begin{aligned}
\lim _{k} \sup \left(N A^{*}\left(x_{n_{k}}\right), A^{*}\left(x_{n_{k}}\right)\right) & \leqq \lim \sup _{n}\left(A N A^{*}\left(x_{n}\right), x_{n}\right) \\
& \leqq(g, x)=(A(f), x)=\left(f, A^{*}(x)\right) .
\end{aligned}
$$

We have the situation where $A^{*}\left(x_{n_{k}}\right) \rightarrow A^{*}(x), N A^{*}\left(x_{n_{k}}\right) \rightarrow f$, and

$$
\lim \sup _{k}\left(N A^{*}\left(x_{n_{k}}\right), A^{*}\left(x_{n_{k}}\right)\right) \leqq\left(f, A^{*}(x)\right) .
$$

Since $N$ is of type (M), we may conclude that $N A^{*}(x)=f$. Thus $A N A^{*}(x)=A(f)=g$. Hence $A N A^{*}$ is of type (M), since it is clearly continuous from finite-dimensional subspaces of $X$ into $X^{*}$ with the weak topology. Q.E.D.

An immediate consequence of Proposition 1.2 and Proposition 1.4 is the following existence theorem for the abstract Hammerstein equation (1.1). In what follows $B(0, r)$ denotes an open ball and $\bar{B}(0, r)$ and $\dot{B}(0, r)$ its closure and boundary, respectively.

THEOREM 1. Let $X$ be a reflexive Banach space and $A: X \rightarrow X^{*}$ a bounded linear map such that the functional $f: X \rightarrow R$ defined by $f(x)=(A(x), x), x \in X$, is weakly lower semicontinuous. Suppose $N: X^{*} \rightarrow X$ is bounded and of type (M). If there exists a number $r>0$ such that

$$
\left(A^{*}(x), x\right)+\left(N A^{*}(x), A^{*}(x)\right) \geqq 0 \text { for all } x \in \dot{B}(0, r),
$$

then there exists an element $w \in X^{*}$ which satisfies equation (1.1).

The following two useful corollaries result from Theorem 1.

Corollary 1.1. Let $X$ be a reflexive Banach space and $N: X^{*} \rightarrow X$ a bounded map of type (M). Suppose $L: X \rightarrow X^{*}$ is linear and monotone, and $C: X \rightarrow X^{*}$ is linear and completely continuous. If there exists a number $r>0$ such that (1.3) holds with $A=L+C$, then the equation

$$
x+(L+C) N(x)=0 \quad\left(x \in X^{*}\right)
$$

has a solution in $X^{*}$. 
Proof. It suffices to show that $A=L+C$ is bounded and $f: X \rightarrow R$ defined by $f(x)=((L+C)(x), x), x \in X$, is a weakly lower semicontinuous functional.

Now, since $L$ is linear and monotone, it follows from the results in [6], [2] that it is bounded. Hence $A$ is bounded. But $L$ is weakly continuous, and thus by Remark 1.1 the functional $x \mapsto(L(x), x)$ is weakly lower semicontinuous.

We will now show that $f$ is weakly lower semicontinuous. Let $\left\{z_{n}\right\} \subset X$ be such that $z_{n} \rightarrow z$ in $X$. Then $C\left(z_{n}\right) \rightarrow C(z)$ and using the initial observation we see that $(L(z), z) \leqq \lim \inf _{n}\left(L\left(z_{n}\right), z_{n}\right)$ and, therefore,

$$
\begin{aligned}
\lim _{n} \inf \left(A\left(z_{n}\right), z_{n}\right) & \geqq \lim _{n} \inf \left(L\left(z_{n}\right), z_{n}\right)+\lim _{n} \inf \left(C\left(z_{n}\right), z_{n}\right) \\
& \geqq(A(z), z),
\end{aligned}
$$

i.e., $f$ is weakly lower semicontinuous. Q.E.D.

REMARK 1.3. In case $C=0$, then $A$ is linear monotone and condition (1.3) of Theorem 1 is satisfied if we assume that $\left(N A^{*}(x), A^{*}(x)\right) \geqq 0$ for all $x \in \dot{B}(0, r) \subset X$. In this case, our Corollary 1.1 is analogous to the result of Brezis [6] for the equation (1.4) with $A=L$. We note that Brezis' arguments are quite different from those of ours. However, in applications his "boundary condition" is more easily verifiable than our condition (1.3).

Corollary 1.2. Let $X$ and $N$ be as in Theorem 1, and suppose $A: X \rightarrow X^{*}$ is linear monotone and such that

$$
\left\|A^{*} x\right\| \geqq \alpha\|x\| \text { for all } x \text { in } X \text { and some } \alpha>0 \text {. }
$$

Suppose further that $N$ is coercive, i.e.,

$$
(N u, u) /\|u\| \rightarrow \infty \text { as }\|u\| \rightarrow \infty .
$$

Then equation (1.1) has a solution in $X^{*}$.

Proof. As the proof of Corollary 1.1 shows, the functional $f: X \rightarrow R$ defined by $f(x)=(A(x), x), x \in X$, is weakly lower semicontinuous. But, since $N$ is coercive, we may choose $r>0$ such that $(N(y), y)>0$ for $\|y\|>r$. Hence, when $\|x\|>r / \alpha$, we have $\left(N A^{*}(x), A^{*}(x)\right)>0$, and since $\left(A^{*}(x), x\right) \geqq 0$ for all $x$ in $X$, we see that condition (1.3) is verified for $x$ such that $\|x\|=r / \alpha$. Q.E.D.

REMARK 1.4. We have shown that $f: X \rightarrow R$ defined by $f(x)=(A(x), x)$ is weakly lower semicontinuous when $A$ is either linear and monotone or the sum of a linear monotone and linear completely continuous mappings. The latter class includes the case studied by Valnberg [22] when $X$ is a Hilbert space $H$ and $A: H \rightarrow H$ is selfadjoint and such that the negative part of its spectrum consists of a finite number of eigenvalues, each of finite multiplicity, while its positive spectrum is arbitrary. In this case $A$ is expressible as $A=A_{+}+A_{-}$, where $A_{+}$is positive selfadjoint and $A_{-}$has a finite-dimensional range, and hence is completely continuous. 
The practical disadvantage of Theorem 1 and Corollary 1.1 is that the verification of its boundary condition (1.3) requires the advance knowledge of $A^{*} x$ for each $x$ in $\dot{B}(0, r) \subset X$ which in some cases may not be easily available. The condition on $A^{*}$ in Corollary 1.2 is also restrictive although not without practical interest.

In this section we show that by strengthening somewhat our conditions on the linear mapping $A$ and by employing the approach of Browder-Gupta [4] (see also Amann [1]), we can replace the boundary condition (1.3) of Theorem 1 by a condition on $N$ which will be more useful for applications.

DEFINITION 2. Let $X$ be a general real Banach space. If $A$ is a linear monotone mapping of $X$ into $X^{*}$, then it is said to be angle-bounded with constant of angleboundedness $c \geqq 0$ if for all $x$ and $y$ in $X$ we have

$$
|(A x, y)-(A y, x)| \leqq 2 c\left\{(A x, x)^{1 / 2}\right\}\left\{(A y, y)^{1 / 2}\right\} .
$$

We recall that if $A$ is a bounded linear mapping of $X$ into $X^{*}$, then $A$ is said to be symmetric if

$$
(A x, y)=(A y, x) \text { for all } x \text { and } y \text { in } X \text {; }
$$

$A$ is strongly accretive (strongly monotone or positive definite) if there exists a constant $m>0$ such that

$$
(A x, x) \geqq m\|x\|^{2} \text { for all } x \text { in } X .
$$

It follows that every symmetric mapping $A$ of $X$ into $X^{*}$ is angle-bounded with constant of angle-boundedness $c=0$. If $A$ is strongly accretive, then it is also angle-bounded with $c=\|A\| / m$ since

$$
|(A x, y)-(A y, x)| \leqq 2\|A\|\|x\|\|y\| \leqq 2 c\left\{(A x, x)^{1 / 2}\right\}\left\{(A y, y)^{1 / 2}\right\}
$$

for all $x, y \in X$.

The following proposition of Browder-Gupta [4] enables one to transform the equation (1.1), i.e., $w+A N w=0$ with $A: X \rightarrow X^{*}$ and $N: X^{*} \rightarrow X$, into an equivalent equation in a suitable Hilbert space $H$.

Proposition 1.5. Let $X$ be a general real Banach space and let $A: X \rightarrow X^{*}$ be angle-bounded. Then there exists a Hilbert space $H$, a continuous linear mapping $S: X \rightarrow H$ with $S^{*}: H \rightarrow X^{*}$ injective, and a bounded skew-symmetric mapping $B: H \rightarrow H$ such that $A=S^{*}(I+B) S$. Furthermore, the following inequalities are valid:

(i) $\|B\| \leqq c$, where $c$ is the constant of angle-boundedness.

(ii) $\|S\|^{2} \leqq R$ if and only if $(A x, x) \leqq R\|x\|^{2}$ for all $x$ in $X$.

Remark 1.5. If $A$ is symmetric, then $c=0$ and hence $B=0$ and $A=S^{*} S$.

In what follows we shall make use of the following known proposition.

Proposition 1.6. If $H$ is a Hilbert space and $B: H \rightarrow H$ a bounded skewsymmetric operator, then $I+B$ is a one-to-one mapping of $H$ onto $H$ such that

$$
\left[(I+B)^{-1} x, x\right] \geqq\left(1+\|B\|^{2}\right)^{-1}|x|^{2} \text { for all } x \text { in } H,
$$

where [, ], | denote the inner product and norm in $H$, respectively. 
In view of Proposition 1.5, it is easy to see that the abstract Hammerstein equation (1.1) has a solution $w$ in $X^{*}$ if and only if the equation

$$
(I+B)^{-1} x+S N S^{*} x=0 \quad(x \in H)
$$

has a solution $x$ in $H$. Thus, it suffices to solve equation (1.11). To do this we need the following proposition:

Proposition 1.7. Let $X$ be a real reflexive Banach space and $A$ an angle-bounded mapping of $X$ into $X^{*}$. If $N$ is a bounded mapping of $X^{*}$ into $X$ of type (M), then the mapping $T \equiv(I+B)^{-1}+S N S^{*}$ of $H$ into $H$ is also of type $(\mathrm{M})$ and bounded.

Proof. Let $\left\{x_{n}\right\} \subset H$ be any sequence such that $x_{n} \rightarrow x_{0}$ in $H, T x_{n} \rightarrow g$ in $H$ and $\lim \sup _{n}\left(T x_{n}, x_{n}\right) \leqq(g, x)$. We wish to show that $T x_{0}=g$. Since $S^{*}$ is a bounded linear mapping of $H$ into $X^{*}$ and $N$ is a bounded map of $X^{*}$ into $X$, it follows that $S^{*} x_{n} \rightarrow S^{*} x_{0}$ in $X^{*}$ and the the sequence $\left\{N S^{*} x_{n}\right\}$ is bounded in $X$. Since $X$ is reflexive, we may choose a subsequence $\left\{x_{n_{k}}\right\}$ and an element $f$ in $X$ such that $N S^{*} x_{n_{k}} \rightarrow f$ in $X$ as $k \rightarrow \infty$. Hence $S N S^{*} x_{n_{k}} \rightarrow S f$ in $H$. But, since $T x_{n} \rightarrow g$ in $H$, we also have $S N S^{*} x_{n_{k}}=T x_{n_{k}}-(I+B)^{-1} x_{n_{k}} \rightarrow g-(I+B)^{-1} x_{0}$ in $H$ and consequently, $S f=g-(I+B)^{-1} x_{0}$. Now, as in the proof of Corollary 1.1, we see that since $(I+B)^{-1}$ is linear and monotone we may conclude that

$$
\lim _{k} \inf \left[(1+B)^{-1} x_{n_{k}}, x_{n_{k}}\right] \geqq\left[(I+B)^{-1} x_{0}, x_{0}\right] .
$$

This, the structure of $T$ and the properties of "lim sup" imply that

$$
\begin{aligned}
\limsup _{k}\left(N S^{*} x_{n_{k}}, S^{*} x_{n_{k}}\right) & =\lim _{k} \sup \left[S N S^{*} x_{n_{k}}, x_{n_{k}}\right] \\
& =\limsup _{k}\left[T x_{n_{k}}-(1+B)^{-1} x_{n_{k}}, x_{n_{k}}\right] \\
& \leqq \limsup _{n}\left[T x_{n}, x_{n}\right]-\liminf _{k}\left[(1+B)^{-1} x_{n_{k}}, x_{n_{k}}\right] \\
& \leqq\left[g-(I+B)^{-1} x_{0}, x_{0}\right]=\left[S f, x_{0}\right]=\left(f, S^{*} x_{0}\right) .
\end{aligned}
$$

Since $N$ is of type (M) and $S^{*} x_{n_{k}} \rightarrow S^{*} x_{0}$ with $N S^{*} x_{n_{k}} \rightarrow f$ in $X$, the relation (+) implies that $N S^{*} x_{0}=f$. Thus, $S N S^{*} x_{0}=S f$ and since $S f=g-(I+B)^{-1} x_{0}$, we obtain the looked for equality $T x_{0}=(1+B)^{-1} x_{0}+S N S^{*} x_{0}=g$, i.e. $T$ is of type (M). Clearly $T$ is bounded. Q.E.D.

THEOREM 2. Let $X$ be a reflexive Banach space, $A$ an angle-bounded mapping of $X$ into $X^{*}$ and $N$ a bounded mapping of type (M) of $X^{*}$ into $X$ such that

$$
(N u, u) \geqq-k\|u\|^{2}+(N(0), u) \text { for all } u \text { in } X^{*} \text { and some } k \geqq 0
$$

with $k$ satisfying the condition $k\left(1+c^{2}\right)\|A\|<1$, where $c$ is the constant of angleboundedness of $A$. Then the equation $w+A N w=0$ has a solution in $X^{*}$.

Proof. In view of the remarks preceding the statement of Proposition 1.7, it suffices to show that equation (1.11) has a solution in $H$, i.e., that $T(x)=0$ has a solution in $H$. Since, by Proposition 1.7, $T$ is a bounded mapping of $H$ into $H$ of 
type (M), in view of Proposition 1.2, it suffices to show that there exists a number $r>0$ such that $[T x, x]>0$ for all $x$ in $\dot{B}(0, r) \subset H$. This, however, follows from the structure of $T,(1.10)$ of Proposition 1.6, Proposition 1.5 and the conditions of Theorem 2 since

$$
\begin{aligned}
{[T x, x] } & =\left[(1+B)^{-1} x, x\right]+\left[S N S^{*} x, x\right]=\left[(1+B)^{-1} x, x\right]+\left(N S^{*} x, S^{*} x\right) \\
& \geqq\left(1+c^{2}\right)^{-1}|x|^{2}-k\left\|S^{*} x\right\|^{2}+\left(N(0), S^{*} x\right) \\
& \geqq\left(c_{0}|x|-\|N(0)\|\|A\|^{1 / 2}\right)|x|,
\end{aligned}
$$

where $c_{0}=\left(1+c^{2}\right)^{-1}-k\|A\|>0$. Hence, there exists a ball $B(0, r)$ such that $c_{0}|x|-\|N(0)\|\|A\|^{1 / 2}>0$ for $x \in \dot{B}(0, r)$ and, hence, $[T x, x]>0$ for all $x \in \dot{B}(0, r)$. Q.E.D.

Theorem 3. Let $X$ be a reflexive Banach space, $A: X \rightarrow X^{*}$ angle-bounded with constant of angle-boundedness $c \geqq 0$, and $N: X^{*} \rightarrow X$ bounded and of type (M) and such that there exist constants $r>0$ and $\gamma>0$ with the property

$$
\|N u\| \leqq \gamma\|u\| \text { for all } u \text { in } X^{*} \text { such that }\|u\| \geqq r,
$$

where $\gamma\left(1+c^{2}\right)\|A\|<1$. Then the equation $w+A N w=0$ has a solution in $X^{*}$.

Proof. Again using the notation of Proposition 1.5 we may conclude, by Proposition 1.7 , that $T=(I+B)^{-1}+S N S^{*}: H \rightarrow H$ is of type (M) and bounded. We now claim that $|[T x, x]| /|x| \rightarrow \infty$ as $|x| \rightarrow \infty$. If this were not the case, then we could choose a sequence $\left\{x_{n}\right\} \subset H$ such that $\left|\left[T x_{n}, x_{n}\right]\right| /\left|x_{n}\right|<M$ for all $n \in N$ and $\left|x_{n}\right| \rightarrow \infty$. We may examine two cases.

Case 1. There exists a subsequence $\left\{x_{n_{k}}\right\}$ such that $\left\|S^{*}\left(x_{n_{k}}\right)\right\|<r$ for all $k$. Hence $\left\{S N S^{*} x_{n_{k}}\right\}$ is bounded and thus $\left\{\left[S N S^{*} x_{n_{k}}, x_{n_{k}}\right] /\left|x_{n_{k}}\right|\right\}$ is also bounded. But we have

$$
\left|\frac{\left[T x_{n_{k}}, x_{n_{k}}\right]}{\left|x_{n_{k}}\right|}\right| \geqq \frac{1}{1+c^{2}}\left|x_{n_{k}}\right|-\left|\frac{\left[S N S^{*} x_{n_{k}}, x_{n_{k}}\right]}{\left|x_{n_{k}}\right|}\right| \rightarrow \infty
$$

as $k \rightarrow \infty$. This is a contradiction.

Case 2. There exists an integer $n_{0} \geqq 1$ such that $\left\|S^{*} x_{n}\right\|>r$ for all $n \geqq n_{0}$. Then for all $n \geqq n_{0}$

$$
\left|\frac{\left[T x_{n}, x_{n}\right]}{\left|x_{n}\right|}\right| \geqq \frac{1}{1+c^{2}}\left|x_{n}\right|-\left|S N S^{*} x_{n}\right| \geqq\left(\frac{1}{1+c^{2}}-\gamma\|A\|\right)\left|x_{n}\right| .
$$

Thus $\left|\left[T x_{n}, x_{n}\right] /\right| x_{n}|| \rightarrow \infty$ as $n \rightarrow \infty$. This again is a contradiction.

Since either Case 1 or Case 2 occurs, we see that our contradictory hypothesis is untenable. Thus, $|[T(x), x]| /|x| \rightarrow \infty$ as $|x| \rightarrow \infty$. Hence there exists $r>0$ such that $[T x, x] \neq 0$ for all $x \in H$ such that $\|x\|>r$. Since $T$ is bounded and of type (M) we may conclude, by Proposition 1.3, that the equation $T x=0$ has a solution in $H$. Hence the equation $w+A N w=0$ has a solution in $X^{*}$. Q.E.D.

REMARK 1.6. Theorems 1,2 and 3 and Corollaries 1.1 and 1.2 are valid when $N$ is bounded, satisfies the respective growth conditions and has any one of the following forms: 
(a) $N=M$, where $M$ is a hemicontinuous monotone map of $X^{*} \rightarrow X$. (We recall that hemicontinuous means continuous from line segments in $X$ to weak topology of $X^{*}$.)

(b) $N=M+S+C$, where $M$ is pseudo-monotone, $S$ is weakly continuous and monotone, and $C$ is completely continuous.

2. In this section we study the abstract Hammerstein equation (1) under the assumption that $X$ is a general Banach space, $A$ is angle-bounded, and $N$ is pseudomonotone.

Definition 3. Let $X$ be a general real Banach space. A mapping $T$ of $X$ into $X^{*}$ is said to be pseudo-monotone if the following two conditions are satisfied:

(i) If $\left\{x_{n}\right\} \subset X$ is a sequence such that $x_{n} \rightarrow x_{0}$ in $X$ and $\lim \sup _{n}\left(T x_{n}, x_{n}-x_{0}\right) \leqq 0$, then

$$
\left(T x_{0}, x_{0}-x\right) \leqq \lim \inf \left(T x_{n}, x_{n}-x\right) \text { for all } x \text { in } X .
$$

(ii) For each fixed $v \in X$, the mappings defined by $x \mapsto(T x, x-v)$ is bounded below on weakly compact subsets of $X$.

The definition of pseudo-monotone map given by Brezis differs from the above in the same manner as the two definitions of a map of type (M) differ.

It was shown in [6] that every pseudo-monotone mapping is of type (M), but that there are mappings of type (M) which are not pseudo-monotone. The same is true for pseudo-monotone as defined above.

REMARK 2.1. It turns out that every mapping $T$ of $X$ into $X^{*}$ which satisfies condition (ii) also satisfies condition $(\beta)$ of Proposition 1.2. Indeed, let $\left\{x_{j}\right\}$ be a bounded sequence in $X$ such that $\left\{\left(T x_{j}, x_{j}\right)\right\}$ is also bounded, i.e., there exist $r>0$ and $K>0$ such that $\left\{x_{j}\right\} \subset B(0, r)$ and $\left|\left(T x_{j}, x_{j}\right)\right| \leqq K$ for all $j$. Now, suppose that $T$ satisfies condition (ii). Then to each $v$ in $X$, there corresponds a $C_{v}(B)>0$ such that

$$
\left(T x_{j}, x_{j}-v\right) \geqq-C_{v}(B) \text { or }\left(T x_{j}, v\right) \leqq K+C_{v}(B) .
$$

Similarly, there exists a $C_{-v}(B)>0$ such that

$$
\left(T x_{j}, x_{j}+v\right) \geqq-C_{-v}(B) \text { or }\left(T x_{j}, v\right) \geqq-K-C_{-v}(B) .
$$

The two inequalities imply that to each $v$ in $X$ there corresponds an $M_{v}>0$ such that $\left|\left(T x_{j}, v\right)\right| \leqq M_{v}$. Hence, by the principle of uniform boundedness, there exists an $m>0$ such that $\left\|T x_{j}\right\| \leqq m$ for all $j$, i.e., condition $(\beta)$ holds.

We note that, in view of the above remarks, Proposition 1.2 implies the following generalization of the result of Brezis [6].

If $X$ is a reflexive Banach space and $T$ is a psuedo-monotone mapping of $X$ into $X^{*}$ such that

$$
(T x, x) /\|x\| \rightarrow+\infty \quad \text { as }\|x\| \rightarrow \infty,
$$

then $T$ is surjective, i.e. $T(X)=X^{*}$. 
The authors were informed by D. G. DeFigueiredo that a similar result has also been recently obtained by F. E. Browder and P. Hess in a paper quoted before the statement of Proposition 1.3.

We add that every hemicontinuous monotone mapping is pseudo-monotone and so is the sum of a pseudo-monotone and a hemicontinuous monotone mappings.

In this section we obtain existence results for the Hammerstein equation (1) without the assumption that $X$ is reflexive, and, in case $N$ is pseudo-monotone, without the assumption that it is bounded. Consequently, the results of this section cannot be deduced from those of the preceding section.

Our first result in this section is the following proposition:

Proposition 2.1. Let $X$ be a general Banach space, $A: X \rightarrow X^{*}$ angle-bounded and $N: X^{*} \rightarrow X$ pseudo-monotone. Then (using the notation of Proposition 1.5) the mapping $T: H \rightarrow H$ defined by $T=(1+B)^{-1}+S N S^{*}$ is pseudo-monotone.

Proof. Let $\left\{x_{n}\right\} \subset H$ be a sequence such that $x_{n} \rightarrow x_{0}$ in $H$ and lim $\sup \left[T x_{n}, x_{n}-x_{0}\right]$ $\leqq 0$. We wish to show that

$$
\left[T x_{0}, x_{0}-x\right] \leqq \lim _{n} \inf \left[T x_{n}, x_{n}-x\right] \text { for all } x \text { in } H \text {. }
$$

Since $x_{n} \rightarrow x_{0}$ we have $(I+B)^{-1} x_{n} \rightarrow(I+B)^{-1} x_{0}$ in $H$ and because $(I+B)^{-1}$ is linear and monotone in $H$, we also have

$$
\left[(I+B)^{-1} x_{0}, x_{0}\right] \leqq \lim _{n} \inf \left[(I+B)^{-1} x_{n}, x_{n}\right] .
$$

On the other hand, for each $x$ in $H,\left[(I+B)^{-1} x_{n},-x\right] \rightarrow\left[(I+B)^{-1} x_{0},-x\right]$ and, therefore, for all $x$ in $H$ we have

$$
\begin{aligned}
{\left[(1+B)^{-1} x_{0}, x_{0}-x\right] } & \leqq \lim _{n} \inf \left[(I+B)^{-1} x_{n}, x_{n}\right]+\lim _{n} \inf \left[(I+B)^{-1} x_{n},-x\right] \\
& \leqq \lim \inf \left[(1+B)^{-1} x_{n}, x_{n}-x\right] .
\end{aligned}
$$

The relation $(\dagger)$ and the structure of $T$ imply that

$$
\begin{aligned}
\lim _{n} \sup \left(N S^{*} x_{n}, S^{*} x_{n}-S^{*} x_{0}\right)= & \lim \sup \left[S N S^{*} x_{n}, x_{n}-x_{0}\right] \\
= & \lim \sup \left[T x_{n}-(1+B)^{-1} x_{n}, x_{n}-x_{0}\right] \\
\leqq & \lim \sup \left[T x_{n}, x_{n}-x_{0}\right] \\
& -\lim \inf \left[(1+B)^{-1} x_{n}, x_{n}-x_{0}\right] .
\end{aligned}
$$

Since $(\dagger)$ holds for all $x$ in $H$, it holds, in particular, for $x=x_{0}$. Hence we may conclude that $\lim \inf _{n}\left[(1+B)^{-1} x_{n}, x_{n}-x_{0}\right] \geqq 0$. This and (f†) imply that

$$
\lim _{n} \sup \left(N S^{*} x_{n}, S^{*} x_{n}-S^{*} x_{0}\right) \leqq 0 \text {. }
$$

But $S^{*} x_{n} \rightarrow S^{*} x_{0}$ in $X^{*}$ and $N$ is a pseudo-monotone mapping of $X^{*}$ into $X$; consequently,

$$
\left(N S^{*} x_{0}, S^{*} x_{0}-v\right) \leqq \lim \inf \left(N S^{*} x_{n}, S^{*} x_{n}-v\right) \text { for all } v \text { in } X^{*}
$$


In particular, the above holds for elements $v$ in $X^{*}$ of the form $v=S^{*} x$ with $x$ in $H$ and, therefore,

$$
\left[S N S^{*} x_{0}, x_{0}-x\right] \leqq \lim _{n} \inf \left[S N S^{*} x_{n}, x_{n}-x\right] \text { for all } x \text { in } H .
$$

Since we have also shown that

$$
\left[(1+B)^{-1} x_{0}, x_{0}-x\right] \leqq \lim _{n} \inf \left[(1+B)^{-1} x_{n}, x_{n}-x\right] \text { for all } x \text { in } H,
$$

it follows from the above two inequalities that

$$
\left[T x_{0}, x_{0}-x\right] \leqq \lim _{n} \inf \left[T x_{n}, x_{n}-x\right] \text { for all } x \text { in } H \text {. }
$$

Next we have to show that if $N: X^{*} \rightarrow X$ satisfies condition (ii) of Definition 3, then so does $T: H \rightarrow H$, i.e., to each fixed $v$ in $H$ and $B(0, r) \subset H$ there corresponds a $C_{v}(B)>0$ such that $[T x, x-v] \geqq-C_{v}(B)$. Now, let $v$ be any given point in $H$ and $x$ any point in $B(0, r)$. Then

$$
\begin{aligned}
{[T x, x-v] } & =\left[(I+B)^{-1} x, x-v\right]+\left[S N S^{*} x, x-v\right] \\
& =\left[(I+B)^{-1} x, x\right]-\left[(I+B)^{-1} x, v\right]+\left(N S^{*} x, S^{*} x-S^{*} v\right) \\
& \geqq\left(1+\|B\|^{2}\right)^{-1}|x|^{2}-\left|(I+B)^{-1}\right||x||v|+\left(N S^{*} x, S^{*} x-S^{*} v\right) .
\end{aligned}
$$

Now, if $x \in B(0, r)$, then $S^{*} x \in B^{*}$ with $B^{*}=S^{*}(B(0, r))$, which is weakly precompact and therefore, by condition (ii) satisfied by $N$, we have

$$
\left(N S^{*} x, S^{*} x-S^{*} v\right) \geqq-C_{v^{*}}\left(B^{*}\right) \quad \text { with } v^{*}=S^{*} v
$$

Since $S^{*}$ is a fixed bounded linear mapping, $C_{v^{*}}\left(B^{*}\right)$ depends only on $r$ and $v$, i.e., we have $C_{v^{*}}\left(B^{*}\right)=C_{v}^{0}(B)$ for some $C_{v}^{0}(B)>0$ depending on $v$ and $B$. The above remarks and $(\pi)$ imply the existence of $C_{v}(B)>0$ such that $[T x, x-v] \geqq-C_{v}(B)$ for each given $v$ in $H$ and $B(0, r) \subset H$. Thus, $T$ is pseudo-monotone. Q.E.D.

Since a pseudo-monotone mapping is of type (M) and satisfies condition $(\beta)$, Propositions 1.2 and 2.1 imply the validity of the following theorems.

THEOREM 4. Let $X$ be a general Banach space, $A$ an angle-bounded mapping of $X$ into $X^{*}$ and $N$ a pseudo-monotone mapping of $X^{*}$ into $X$ such that

$$
(N u, u) \geqq-k\|u\|^{2}+(N(0), u) \text { for all } u \text { in } X^{*} \text { and some } k \geqq 0
$$

with $k$ satisfying the relation $k\left(1+c^{2}\right) R<1$, then the equation $w+A N w=0$ has a solution in $X^{*}$.

Proof. By Proposition 2.1, $T: H \rightarrow H$ is pseudo-monotone and hence satisfies condition $(\beta)$ and is of type (M). Moreover, the same arguments as those used in the proof of Theorem 2 show that there exists $r>0$ such that $[T x, x]>0$ for all $x \in \dot{B}(0, r) \subset H$. Hence, by Proposition $1.2, T x=0$ has a solution in $B(0, r)$ and, therefore, equation (1.1) is solvable in $X^{*}$. Q.E.D. 
Theorem 5. Let $X$ be a general Banach space and $A: X \rightarrow X^{*}$ angle-bounded with constant of angle-boundedness $c \geqq 0$. Suppose $N: X^{*} \rightarrow X$ is pseudo-monotone bounded and such that there exist constants $r>0$ and $\gamma>0$ such that $\|N u\| \leqq \gamma\|u\|$ for all $u$ in $X^{*}$ such that $\|u\|>r$, where $\gamma\left(1 \pm c^{2}\right)\|A\|<1$. Then the equation $w+A N w=0$ has a solution in $X^{*}$.

Proof. Once more using the notation of Proposition 1.5 we conclude from Proposition 2.1 that $T=(I+B)^{-1}+S N S^{*}: H \rightarrow H$ is pseudo-monotone and bounded. Hence it is of type (M) and bounded. By an argument precisely the same as that in Theorem 3 one concludes that $|[T(x), x]| /|x| \rightarrow \infty$ as $|x| \rightarrow \infty$. Hence we may find $r^{\prime}>0$ such that $[T(x), x] \neq 0$ for all $x$ such that $|x|>r^{\prime}$, and by applying Proposition 1.3 conclude that the equation $T(x)=0$ has a solution in $H$. Thus $u+A N(u)=0$ has a solution in $X^{*}$. Q.E.D.

3. In Theorems 2 and 3 we assumed that the Banach space $X$ was reflexive and then utilized Proposition 1.5 in applying existence theorems for mappings of type (M). In fact, if one is willing to assume a slightly stronger hypothesis on $A$ than angle-boundedness, then one may relax the reflexivity condition to that of weak completeness on $X$.

Theorem 6. Let $X$ be a weakly complete Banach space. Suppose $A: X \rightarrow X^{*}$ is angle-bounded and such that $(A x, x)>0$ for $x \neq 0$. Suppose $N: X^{*} \rightarrow X$ is bounded and of type (M), and furthermore suppose that there exists $\gamma>0$ and $r>0$ such that $\|N(u)\| \leqq \gamma\|u\|$ for all $u \in X^{*}$ with $\|u\|>r$, where $\gamma\left(1+c^{2}\right)\|A\|<1$. Then the equation $w+A N(w)=f$ has a solution in $X^{*}$ for all $f$ lying in a dense subspace of $X^{*}$.

Proof. We shall first of all show that the bounded mapping

$$
T=(I+B)^{-1}+S N S^{*}: H \rightarrow H
$$

is of type (M), where $S, B$ and $H$ are as in Proposition 1.5.

Suppose $\left\{x_{n}\right\} \subset H$ is such that $x_{n} \rightarrow x_{0}, T\left(x_{n}\right) \rightarrow g$ and $\lim \sup _{n}\left[T\left(x_{n}\right), x_{n}\right]$ $\leqq\left[g, x_{0}\right]$. Now $\left\{x_{n}\right\}$ is bounded and hence $\left\{S^{*}\left(x_{n}\right)\right\}$ is bounded, and hence $\left\{S N S^{*}\left(x_{n}\right)\right\}$ is bounded. Since $H$ is reflexive, we may choose $\left\{x_{n_{k}}\right\}$ such that $S N S^{*}\left(x_{n_{k}}\right) \rightarrow g$ in $H$. Hence for all $h \in H$ we have $\left[S N S^{*}\left(x_{n_{k}}\right), h\right] \rightarrow[g, h]$, i.e., for all $h \in H,\left(N S^{*}\left(x_{n_{k}}\right), S^{*}(h)\right) \rightarrow[g, h]$. But observe that since $|S(u)|^{2}=(A(u), u)$ we may conclude that $S: X \rightarrow H$ is one-to-one and thus $S^{*}(H)$ is dense in $X^{*}$. Consequently, for a dense subset of $X^{*}$, namely $S^{*}(H)$, we have $\left\{\left(N S^{*}\left(x_{n_{k}}\right), g\right)\right\}$ is Cauchy for all $g$ in $S^{*}(H)$. Since $\left\{N S^{*}\left(x_{n_{k}}\right)\right\}$ is bounded we have that $\left\{\left(N S^{*}\left(x_{n_{k}}\right), g\right)\right\}$ is Cauchy $\forall g \in X^{*}$. Since $X$ is weakly complete, we may choose $f \in X$ such that $N S^{*}\left(x_{n_{k}}\right) \rightarrow f$. Hence $S N S^{*}\left(x_{n_{k}}\right) \rightarrow S(f)$. But since

$$
(I+B)^{-1}\left(x_{n_{k}}\right) \rightarrow(I+B)^{-1}\left(x_{0}\right),
$$

we see that

$$
S N S^{*}\left(x_{n_{k}}\right)=T\left(x_{n_{k}}\right)-(I+B)^{-1}\left(x_{n_{k}}\right) \rightarrow g-(I+B)^{-1}\left(x_{0}\right) .
$$


Thus, $S(f)=g-(I+B)^{-1}\left(x_{0}\right)$. Now,

$$
\begin{aligned}
\limsup _{k}\left(N S^{*}\left(x_{n_{k}}\right), S^{*}\left(x_{n_{k}}\right)\right) & =\lim _{k} \sup \left[S N S^{*}\left(x_{n_{k}}\right), x_{n_{k}}\right] \\
& =\lim _{k} \sup \left[T\left(x_{n_{k}}\right)-(I+B)^{-1}\left(x_{n_{k}}\right), x_{n_{k}}\right] \\
& \leqq \lim _{n} \sup \left[T\left(x_{n}\right), x_{n}\right]-\liminf _{k}\left[(I+B)^{-1}\left(x_{n_{k}}\right), x_{n_{k}}\right] \\
& \leqq\left[g-(I+B)^{-1}\left(x_{0}\right), x_{0}\right],
\end{aligned}
$$

since $\lim \inf _{k}\left[(I+B)^{-1}\left(x_{n_{k}}\right), x_{n_{k}}\right] \geqq\left[(I+B)^{-1}\left(x_{0}\right), x_{0}\right]$.

Thus, $\lim \sup _{k}\left(N S^{*}\left(x_{n_{k}}\right), S^{*}\left(x_{n_{k}}\right)\right) \leqq\left(f, S^{*}\left(x_{0}\right)\right)$ and $S^{*}\left(x_{n_{k}}\right) \rightarrow S^{*}\left(x_{0}\right)$ and $N S^{*}\left(x_{n_{k}}\right) \rightarrow f$. Since $N$ is of type $(\mathrm{M})$ we see that $N S^{*}\left(x_{0}\right)=f$. Thus $S N S^{*}\left(x_{0}\right)$ $=S(f)=g-(I+B)^{-1}\left(x_{0}\right)$. Finally, we see $T\left(x_{0}\right)=g$, and hence $T$ is of type (M).

The argument of Theorem 3 showing $|[T(x), x]| /|x| \rightarrow \infty$ as $|x| \rightarrow \infty$ is also valid here.

Let $h \in H$, then it follows that $T_{h}=T-h$ is bounded and of type (M) and such that there exists $r>0$ such that $\left[T_{h}(x), x\right] \neq 0$ for all $x \in H$ such that $|x| \geqq r$. By Proposition 1.3, we may conclude that $T_{h}(x)=0$ has a solution. Thus $T$ is onto. Now, since $I+B: H \rightarrow H$ is onto, we see that $S^{*}(I+B)(H)=S^{*}(H)$. Let $f \in S^{*}(I+B)(H)$. Then choose $h_{1} \in H$ such that $f=S^{*}(I+B)\left(h_{1}\right)$. Choose $h_{2} \in H$ such that $T h_{2}=(I+B)^{-1}\left(h_{2}\right)+S N S^{*}\left(h_{2}\right)=h_{1}$. Then $S^{*}\left(h_{2}\right)+A N\left(S^{*}\left(h_{2}\right)\right)=f$. Since $S^{*}(I+B)(H)$ is a dense subset of $X^{*}$, our proof is complete. Q.E.D.

THEOREM 7. Let $X$ be a weakly complete Banach space. Suppose $A: X \rightarrow X^{*}$ is angle-bounded and such that $(A(x), x)>0$ for $x \neq 0$. Let $N: X^{*} \rightarrow X$ be bounded and of type (M). Suppose that $(N(u), u) \geqq-k\|u\|^{2}+(N(0), u)$ for all $u$ in $X^{*}$ and some $k$ such that $k\left(1+c^{2}\right)\|A\|<1$, where $c$ is the constant of angle-boundedness. Then the equation $w+A N(w)=0$ has a solution in $X^{*}$.

Proof. In Theorem 5 we proved that the map $T=(I+B)^{-1}+S N S^{*}: H \rightarrow H$ is of type (M) and bounded. Now

$$
\begin{aligned}
{[T(x), x] } & =\left[(I+B)^{-1}(x), x\right]+\left(N S^{*}(x), S^{*}(x)\right) \\
& \geqq\left(1+c^{2}\right)^{-1}|x|^{2}-k\left\|S^{*}(x)\right\|^{2}+\left(N(0), S^{*}(x)\right) \\
& \geqq\left(c_{0}|x|-\|N(0)\| \cdot\|A\|^{1 / 2}\right)|x|, \quad \text { where } c_{0}=\left(1+c^{2}\right)^{-1}-k\|A\| .
\end{aligned}
$$

Hence there exists $r>0$ such that $[T(x), x]>0$ for all $x$ such that $|x|=r$. Hence, by Proposition 1.2, we may find $x_{0} \in H$ such that $T\left(x_{0}\right)=0$. Hence the equation $w+A N(w)=0$ has a solution. Q.E.D.

If we strengthen further the conditions on $A$ by assuming that $A$ is strongly accretive on $X$, i.e., $(A x, x) \geqq m\|x\|^{2}$ for all $x$ in $X$ and some $m>0$, then Theorem 3 remains valid for the mapping $N: X^{*} \rightarrow X$ of type (M) for which the boundedness assumption is somewhat relaxed.

Proposition 3.1. Let $X$ be a Banach space and let $A$ be a strongly accretive linear mapping of $X$ into $X^{*}$. If $N$ is a mapping of $X^{*}$ into $X$ which is of type (M) and 
which satisfies condition $(\beta)$, then $T=(1+B)^{-1}+S N S^{*}: H \rightarrow H$ is also of type $(\mathrm{M})$ and satisfies condition $(\beta)$.

Proof. We first note that since $A$ is a strongly accretive linear mapping of $X$ into $X^{*}, A$ is angle-bounded with the constant of angle-boundedness $c=\|A\| / m$. Now, if in $X$ we introduce the symmetric bilinear form [ , ] on $X$, by

$$
[x, y]=\frac{1}{2}\{(A x, y)+(A y, x)\} \text { for all } x, y \in X,
$$

then, since $\|A\|\|x\| \geqq[x, x]=|x|^{2}=(A x, x) \geqq m\|x\|^{2}$ for all $x$ in $X$, the space $X$ endowed with the inner product [, ] and the corresponding norm [ ; ] = $\left.\quad\right|^{2}$ becomes a Hilbert space, which we also denote by $H$. We add in passing that since the norms $\|\quad\|$ and $\mid \quad$ are equivalent and $X$ is a Hilbert space with respect to the norm $\mid, X$ is reflexive.

As in [4], let $S$ be the linear injection of $X$ into $H$. Since $|S x|^{2}=[S x, S x]=(A x, x)$ $\geqq m\|x\|^{2}$ for all $x$ in $X, S$ is a continuously invertible mapping of $X$ onto $H$. Consequently, $S^{*}$ is also a continuously invertible mapping of $H$ onto $X^{*}$. Since $A$ is angle-bounded, the same arguments as those used in [4] show that there exists a bounded skew-symmetric operator $B$ of $H$ into $H$ such that $A=S^{*}(I+B) S$.

Note that since $N$ is not bounded we cannot use Proposition 1.7 to conclude that $T=(1+B)^{-1}+S N S^{*}$ is of type (M). For that, a separate argument has to be given.

Now, let $\left\{x_{n}\right\}$ be any sequence in $H$ such that $x_{n} \rightarrow x_{0}$ in $H, T x_{n} \rightarrow g$ in $H$ and $\lim \sup _{n}\left[T x_{n}, x_{n}\right] \leqq\left[g, x_{0}\right]$. Then $S^{*} x_{n} \rightarrow S^{*} x_{0}$ in $X^{*}$ and $(1+B)^{-1} x_{n} \rightarrow(1+B)^{-1} x_{0}$ in $H$. Hence $S N S^{*} x_{n}=T x_{n}-(1+B)^{-1} x_{n} \rightarrow g-(1+B)^{-1} x_{0}$ in $H$ and, therefore, since $S$ is a continuously invertible linear mapping,

$$
N S^{*} x_{n}=S^{-1}\left(S N S^{*} x_{n}\right) \rightarrow S^{-1}\left(g-(1+B)^{-1} x_{0}\right) \text { in } X .
$$

Since $\lim \inf _{n}\left[(1+B)^{-1} x_{n}, x_{n}\right] \geqq\left[(1+B)^{-1} x_{0}, x_{0}\right]$ we have

$$
\begin{aligned}
\lim \sup \left(N S^{*} x_{n}, S^{*} x_{n}\right) & =\limsup _{n}\left[S N S^{*} x_{n}, x_{n}\right]=\lim \sup \left[T x_{n}-(1+B)^{-1} x_{n}, x_{n}\right] \\
& \leqq \lim \sup \left[T x_{n}, x_{n}\right]-\lim \inf \left[(1+B)^{-1} x_{n}, x_{n}\right] \\
& \leqq\left[g, x_{0}\right]-\left[(1+B)^{-1} x_{0}, x_{0}\right]=\left[g-(1+B)^{-1} x_{0}, x_{0}\right] \\
& =\left[S S^{-1}\left(g-(1+B)^{-1} x_{0}, x_{0}\right)\right] \\
& =\left(S^{-1}\left(g-(1+B)^{-1} x_{0}\right), S^{*} x_{0}\right) .
\end{aligned}
$$

Thus, we have $S^{*} x_{n} \rightarrow S^{*} x_{0}$ in $X^{*}, N S^{*} x_{n} \rightarrow S^{-1}\left(g-(1+B)^{-1} x_{0}\right)$ in $X$ and $\lim \sup \left(N S^{*} x_{n}, S^{*} x_{n}\right) \leqq\left(S^{-1}\left(g-(1+B)^{-1} x_{0}\right), S^{*} x_{0}\right)$ from which, since $N$ is of type (M), we conclude that $N S^{*} x_{0}=S^{-1}\left(g-(1+B)^{-1} x_{0}\right)$, i.e. $T x_{0}=g$ and $T$ is of type (M).

To show that $T$ satisfies condition $(\beta)$ let $\left\{x_{j}\right\}$ be a bounded sequence in $H$ such that $\left\{\left[T x_{j}, x_{j}\right]\right\}$ is also bounded. Now, since $\left\{S^{*} x_{j}\right\}$ is bounded in $X^{*}$ and $(I+B)^{-1}$ is a bounded linear mapping of $H$ onto $H$, it follows from the equality

$$
\left(N S^{*} x_{j}, S^{*} x_{j}\right)=\left[S N S^{*} x_{j}, x_{j}\right]=\left[T x_{j}, x_{j}\right]-\left[(I+B)^{-1} x_{j}, x_{j}\right]
$$


and the hypothetical assumptions on $\left\{x_{j}\right\}$ and $\left\{\left[T x_{j}, x_{j}\right]\right\}$ that $\left\{\left(N S^{*} x_{j}, S^{*} x_{j}\right)\right\}$ is bounded. Consequently, by $(\beta)$ satisfied by $N$, the sequence $\left\{N S^{*} x_{j}\right\}$ is bounded in $X$. Hence $\left\{S N S^{*} x_{j}\right\}$ is bounded in $H$. Since $T x_{j}=(I+B)^{-1} x_{j}+S N S^{*} x_{j}$ we see that $\left\{T x_{j}\right\}$ is bounded in $H$, i.e., $T$ satisfies condition $(\beta)$. This completes the proof of Proposition 3.1. Q.E.D.

Now, Propositions 1.2 and 3.1 and the argument analogous to the proof of Theorems 2 or 3 imply the validity of the following theorem.

TheOREM 8. Let $X$ be a Banach space, $A$ a strongly accretive linear mapping of $X$ into $X^{*}$ and $N$ a mapping of type $(\mathrm{M})$ of $X^{*}$ into $X$ such that condition $(\beta)$ holds and

$$
(N u, u) \geqq-k\|u\|^{2}+(N(0), u) \text { for all } u \text { in } X^{*} \text { and some } k \geqq 0
$$

with $k\left(1+c^{2}\right)\|A\|<1$. Then equation (1.1), $w+A N w=0$, has a solution in $X^{*}$.

Similarly, using Proposition 3.1 and arguing as in Theorem 5 we obtain

THEOREM 9. Let $X$ be a Banach space, $A: X \rightarrow X^{*}$ a strongly accretive mapping, and $N: X^{*} \rightarrow X$ of type $(\mathrm{M})$ and such that condition $(\beta)$ holds and there exist $\gamma>0$ and $r>0$ such that

$$
\|N(u)\| \leqq \gamma\|u\| \text { for all } u \text { such that }\|u\| \geqq r,
$$

where $\gamma\left(1+\|A\|^{2} / m^{2}\right)\|A\|<1$ with $m>0$ such that $(A(x), x) \geqq m\|x\|^{2}, x \in X$. Then the equation $w+A N(w)=f$ has a solution for each $f \in X^{*}$.

Proof. Since $S^{*}(I+B)(H)=X^{*}$, to prove Theorem 9 it suffices to show that $T$ is onto. Now, by Proposition 3.1, T:H $\rightarrow H$ is of type (M) and satisfies condition $(\beta)$ and so is the mapping $T_{f}=T-f$ for each given $f$ in $H$. Hence, to prove our assertion, it suffices to show that $0 \in R(T)$. The latter will follow if we can establish the existence of an $r_{1}>0$ such that $[T x, x]>0$ for all $x \in \dot{B}\left(0, r_{1}\right) \subset H$. Now, since $S^{*}: H \rightarrow X^{*}$ is continuously invertible, there exists a $c_{1}>0$ such that $\left\|S^{*} x\right\|$ $\geqq c_{1}\|x\|$ for all $x$ in $H$. Choose $r_{1}>0$ such that $c_{1} r_{1}>r$ and note that for all $\|x\| \geqq r_{1}$ we have $\left\|S^{*} x\right\| \geqq c_{1}\|x\| \geqq c_{1} r_{1}>r$. Hence, in view of our conditions on $N$ and the fact that $\left\|S^{*}\right\|^{2} \leqq\|A\|$ and $\|B\| \leqq\|A\| / m$, for all $\|x\| \geqq r_{1}$ we have the relation

$$
\left|\left(N S^{*} x, S^{*} x\right)\right| \leqq\left\|N S^{*} x\right\|\left\|S^{*} x\right\| \leqq \gamma\left\|S^{*}\right\|^{2}|x|^{2} \leqq \gamma\|A\||x|^{2}
$$

and therefore for all $x$ in $H$ with $|x|>r_{1}$ we have

$$
\begin{aligned}
{[T x, x] } & =\left[(I+B)^{-1} x, x\right]+\left[S N S^{*} x, x\right] \geqq\left(1+\|B\|^{2}\right)^{-1}|x|^{2}-\left|\left(N S^{*} x, S^{*} x\right)\right| \\
& \geqq c|x|^{2},
\end{aligned}
$$

where $c=\left(1+\|B\|^{2}\right)^{-1}-\gamma\|A\|>0$ since $\gamma\left(1+\|A\|^{2} / m^{2}\right)\|A\|<1$ by hypothesis. Q.E.D.

4. Using the results of Proposition 1.5 and Remark 1.5, in this section we extend VaInberg's result [22] to general Banach spaces under the assumption that $A: X \rightarrow X^{*}$ is a linear monotone and symmetric mapping and, thus, anglebounded with constant of angle-boundedness $c=0$. 
In what follows we shall make use of the following known fact.

Proposition 4.1. Let $X$ be a reflexive Banach space and, in particular, a Hilbert space. Let $f: \bar{B}(0, r): X \rightarrow R^{1}$ be a weakly lower semicontinuous functional. Then $f$ assumes its infimum on $\bar{B}(0, r)$. Furthermore, if $f(x)>f(0)$ for all $x \in \dot{B}(0, r)$, then $f$ attains a local minimum at an interior point of $\bar{B}(0, r)$.

We recall that a mapping $G$ of $X$ into $X^{*}$ is said to be potential (weakly potential) if there exists a functional $f$ of $X$ into $R^{1}$ such that for all $x$ and $y$ in $X$ we have

$$
\lim _{t \rightarrow 0} \frac{f(x+t y)-f(x)}{t}=(G x, y) .
$$

The functional $f$ is called the potential of $G$ and $G$ is said to be the gradient of $f$, written $\operatorname{grad}(f)=G$.

REMARK 4.1. When $G$ is potential, then $G x=0$ whenever $x$ is a local minimum or maximum of $f$, where $\operatorname{grad}(f)=G$.

THEOREM 10. Let $X$ be a general Banach space and let $A$ be a linear, monotone, and symmetric mapping of $X$ into $X^{*}$. Suppose $f$ is a weakly lower semicontinuous functional on $X$ such that

$$
f(x) \geqq-\frac{1}{2} a_{1}\|x\|^{2}-a_{2}\|x\|^{\delta}-a_{3}
$$

where $a_{1}\|A\|<1, a_{2}>0, a_{3}>0$ and $0<\delta<2$. Suppose also that $N: X^{*} \rightarrow X$ is such that $\operatorname{grad}(f)=N$. Then the equation $w+A N w=0$ has a solution in $X^{*}$.

Proof. Since, when $A$ is symmetric, $B=0$ and, therefore, in terms of notation of Proposition 1.5, it suffices to find a solution of the equation

$$
x+S N S^{*} x=0, \quad x \in H .
$$

Define the functional $q(x)=\frac{1}{2}[x, x]+f\left(S^{*} x\right)$ for $x \in H$. We note that $q$ is weakly lower semicontinuous. Indeed, suppose $x_{n} \rightarrow x$ in $H$. Then $\frac{1}{2}[x, x]=\frac{1}{2}|x|^{2}$ $\leqq \lim \inf _{n}\left|x_{n}\right|^{2}$ and $S^{*} x_{n} \rightarrow S^{*} x$ in $X^{*}$. Hence, by the weak lower semicontinuity of $f$, we have $f\left(S^{*} x\right) \leqq \lim \inf _{n} f\left(S^{*} x_{n}\right)$. Consequently,

$$
\begin{aligned}
q(x) & =\frac{1}{2}[x, x]+f\left(S^{*} x\right) \leqq \liminf _{n} \frac{1}{2}\left[x_{n}, x_{n}\right]+\liminf _{n} f\left(S^{*} x_{n}\right) \\
& \leqq \liminf _{n}\left\{\frac{1}{2}\left[x_{n}, x_{n}\right]+f\left(S^{*} x_{n}\right)\right\}=\liminf _{n} q\left(x_{n}\right) .
\end{aligned}
$$

Also, using (4.1) and Proposition 1.5, for each $x$ in $H$ we have

$$
\begin{aligned}
q(x) & \geqq \frac{1}{2}|x|^{2}-\frac{1}{2} a_{1}\left\|S^{*} x\right\|^{2}-a_{2}\left\|S^{*} x\right\|^{\delta}-a_{3} \\
& \geqq \frac{1}{2}\left(1-a_{1}\|A\|\right)|\dot{x}|^{2}-a_{2}\|A\|^{\delta / 2}|x|^{\delta}-a_{3} .
\end{aligned}
$$

Hence, we see from our conditions on $a_{1}, a_{2}, a_{3}$ and $\delta$ that $q(x) \rightarrow \infty$ as $|x| \rightarrow \infty$ and, consequently, there exists $r>0$ such that $q(x)>q(0)$ for all $x \in \dot{B}(0, r) \subset H$. Thus, by Proposition 4.1, $q$ has a local minimum at some point $x_{0}$ in $B(0, r)$. But $\operatorname{grad}\left(\frac{1}{2}[x, x]\right)=I$ and $\operatorname{grad}\left(f\left(S^{*} x\right)\right)=S N S^{*}$. Hence, the mapping $I+S N S^{*}: H \rightarrow H$ has a zero, i.e., equation (4.2) is solvable. Q.E.D. 
For the reflexive Banach space $X$ the validity of Theorem 10 has been independently established in [11].

We recall that a mapping $G$ of $X$ into $X^{*}$ is said to have a Gateaux derivative at $x \in X$ if there exists a bounded linear mapping of $X$ into $X^{*}$ denoted by $G^{\prime}(x)$ such that for each $h$ in $X$ we have

$$
\lim _{t \rightarrow 0} \frac{G(x+t h)-G x}{t}=G^{\prime}(x) h .
$$

We say that $G$ is Gateaux differentiable if $G$ has a Gateaux derivative at each $x$ in $X$. Sometimes $G^{\prime}(x)$ is denoted by $D G(x, \cdot)=G^{\prime}(x)$.

While in Theorem 10 we assumed a growth condition on the potential of $N$ to obtain the existence of a solution, in the next theorem we will assume, in addition to the potentialness of $N$, that it has a Gateaux derivative $N^{\prime}$ and place a growth condition on $N^{\prime}$. The following known proposition will be needed.

Proposition 4.2 [22]. Let $X$ be a reflexive Banach space (in particular, a Hilbert space). Suppose $f: X \rightarrow R^{1}$ is such that it has first and second Gateaux derivatives on all of $X$, with the latter satisfying the inequality

$$
D^{2} f(x, h, h) \geqq\|h\| \gamma(\|h\|)
$$

and $D^{2} f(t x, h, h)$ being continuous in $t \in[0,1]$ for $x$ and $h$ fixed, where $\gamma(t)$ is a nonnegative continuous function defined for $t \geqq 0$ and such that $\lim _{t \rightarrow \infty} \gamma(t)=0$. Then there exist $x_{0} \in X$ such that $f$ has a local minimum at $x_{0}$.

TheOREM 11. Let $X$ be a Banach space with $A: X \rightarrow X^{*}$ linear, monotone and symmetric. Let $N: X^{*} \rightarrow X$ be potential and have a Gateaux derivative which satisfies the inequality

$$
D N(x, y, y) \geqq-a\|y\|^{2} \quad\left(y \in X, x \in X^{*}\right)
$$

and $D N(t x, y, y)$ is continuous in $t \in[0,1]$ for $x$ and $y$ fixed, where $a\|A\|<1$. Then the equation $w+A N w=0$ has a solution in $X^{*}$.

Proof. Using the notation of Proposition 1.5, it suffices to find a solution in $H$ to

$$
x+\operatorname{SNS} *(x)=0 .
$$

Define $q(x)=\frac{1}{2}[x, x]+f\left(S^{*}(x)\right)$, where $\operatorname{grad}(f)=N$. Now

$$
D q(x, h)=\lim _{t \rightarrow 0} \frac{q(x+t h)-q(x)}{t}=[x, h]+\left(N S^{*}(x), S^{*}(h)\right)
$$

and

$$
\begin{aligned}
D^{2} q(x, k, h) & =\lim _{t \rightarrow 0} \frac{1}{t}\{D q(x+t k, h)-D q(x, h)\} \\
& =[k, h]+\lim _{t \rightarrow 0} \frac{1}{t}\left\{\left(N\left(S^{*}(x)+t S^{*}(k)\right)-N S^{*}(x)\right), S^{*}(h)\right\} \\
& =[k, h]+D N\left(S^{*}(x), S^{*}(k), S^{*}(h)\right)
\end{aligned}
$$


Hence we have the inequality

$$
D^{2} q(x, h, h) \geqq|h|^{2}-a\|A\||h|^{2} \geqq(1-a\|A\|)|h|^{2} .
$$

We now invoke Proposition 4.2 to conclude that $q$ has a local minimum. Hence $\operatorname{grad}(q)$ has a zero. Thus, there exists $x_{0} \in H$ such that $x_{0}+S N S^{*}\left(x_{0}\right)=0$. Thus there exists $w \in X^{*}$ such that $w+A N(w)=0$. Q.E.D.

We will now remove the symmetry condition on $A$ and replace it with the weaker condition of angle-boundedness. However, in order to obtain the existence of solutions, we shall have to impose, in addition to a growth condition, a "monotonicity" condition on $N$. We do not require $N$ to be potential. We add that our next theorem indicates also the usefulness and the naturalness of our growth condition (1.12) of Theorem 2.

Theorem 12. Let $X$ be a Banach space and suppose $A: X \rightarrow X^{*}$ is angle-bounded. Suppose $N: X^{*} \rightarrow X$ is Gateaux differentiable and its Gateaux derivative satisfies

(+) $\quad D N(x, h, h) \geqq-a\|h\|^{2}, \quad x \in X^{*}, h \in X$, with $a\left(1+c^{2}\right)\|A\|<1$, and $D N(t x, h, h)$ is continuous in $t \in[0,1]$ for $x$ and $h$ fixed.

Suppose furthermore that $N$ satisfies one of the following two conditions:

(i) $N$ is pseudo-monotone,

(ii) $N$ is of type (M) and bounded.

Then there exists $w \in X^{*}$ such that $w+A N(w)=0$.

Proof. By an application of the Fundamental Theorem of Calculus to the function $f: R \rightarrow R$, defined by $f(t)=(N(t x), x)$, one may obtain the following formula

$$
(N(x), x)=(N(0), x)+\int_{0}^{1} D N(t x, x, x) d t
$$

Consequently, applying inequality $(+)$ we see that

$$
(N(x), x) \geqq(N(0), x)-a\|x\|^{2} .
$$

Now, if $N$ satisfies (i) we may invoke Theorem 4 to conclude that $w+A N(w)=0$ has a solution; if $N$ satisfies (ii), then an application of Theorem 2 guarantees a solution. Q.E.D.

REMARK 4.2. Let $\Omega \subset R^{n}$ be measurable with respect to Lebesgue measure. Suppose $k(x, y): \Omega \times \Omega \rightarrow R^{1}$ and $g(u, x): R^{1} \times \Omega \rightarrow R^{1}$. In [11] Hammerstein initiated the study of the following equation:

$$
u(x)+\int_{\Omega} k(x, y) g(u(y), y) d y=0,
$$

where $u(x)$, defined on $\Omega$, is measurable and has certain integrability properties. Letting $A(u)(y)=\int_{\Omega} k(x, y) u(x) d x$ and $N(u)(y)=g(u(y), y)$ we see that the above equation is of the form $u+A N(u)=0$. 
The condition that the operator $N$ has a Gateaux derivative satisfying the growth condition of Theorem 11 is satisfied under the following conditions. Suppose $N$ acts from $L^{p}(\Omega)$ into $L^{q}(\Omega), p^{-1}+q^{-1}=1$. (Nemyckir gives necessary and sufficient conditions for this to be so.) Let $g_{u}^{\prime}(u, x)$ exist and be such that the operator $S$ defined by $S(u)(x)=g_{u}^{\prime}(u(x), x)$ acts from $L^{p}(\Omega)$ into $L^{p /(p-2)}(\Omega)$. Then $N$ has a Gateaux derivative given by $D N(u, v)=g_{u}^{\prime}(u(x), x) \cdot v(x)$. Furthermore, if there exists $m$ such that $g_{u}^{\prime}(u, x) \geqq-m$ for all $u, x$ in $\Omega$, then we have for all $v \in L^{q}(\Omega)$ the relation

$$
\begin{aligned}
D N(u, v, v)=\int_{\Omega} g_{u}^{\prime}(u(x), x) v^{2}(x) d x & \geqq-m\|v\|_{L^{2}(\Omega)}^{2_{2}} \\
& \geqq-m^{1}\|v\|_{L}^{q_{2}(\Omega)}
\end{aligned}
$$

provided $q \geqq 2$ and mes $(\Omega)<\infty$.

REMARK 4.3. The condition that $N$ be potential and bounded is satisfied when $N$, defined by $N(u)(x)=g(u(x), x)$, acts from $L^{p}(\Omega)$ to $L^{q}(\Omega)$ where $p^{-1}+q^{-1}=1$.

REMARK 4.4. Theorem 10 is a generalization of Theorem 10.1 [22], in which it is assumed that all of the operators act in the same Hilbert space. It is also an extension of the result of Vainnberg obtained in [22] for $X=L^{p}(\Omega)(2 \leqq p<\infty)$ with $A$ a completely continuous, monotone, linear and symmetric mapping of $L^{q}(\Omega)$ into $L^{p}(\Omega)(1 / p+1 / q=1)$. Theorem 11 is a generalization of Theorem 10.2 and Theorem 24.3 [22].

5. In case the reflexive space $X$ has a Schauder basis, $X^{*}$ is strictly convex and $A N: X^{*} \rightarrow X^{*}$ is quasibounded, then the first part of $\S 1$ can be somewhat strengthened. To show this we utilize the following result of Petryshyn [19] which was obtained in [19] as a corollary of a more general theorem for pseudo- $A$-proper mappings from $X$ into $Y$.

Proposition 5.1. Let $X$ be a reflexive Banach space with a Schauder basis and with $X^{*}$ strictly convex. Let $T$ be a mapping of $X$ into $X^{*}$ such that

$$
\begin{gathered}
(T x, x) \geqq(T(0), x)-c\|x\| \quad \text { for all } x \text { in } X \text { and some } c \geqq 0, \\
\|T x\| \rightarrow \infty \text { as }\|x\| \rightarrow \infty
\end{gathered}
$$

Then $T$ maps $X$ onto $X^{*}$ provided $T$ satisfies additionally any one of the following three conditions:

(a) $T$ is hemicontinuous and monotone.

(b) $T$ is bounded and pseudo-monotone.

(c) $T$ is bounded and of type (M) and $X$ has a weakly continuous duality mapping $J$ of $X$ into $X^{*}$.

REMARK 5.1. We note in passing that in case $T$ is monotone, then condition (5.1) is superfluous since it always holds with $c=0$.

In what follows we will need a special case of the following proposition. 
Proposition 5.2. Let $X$ be a reflexive Banach space and let $A$ be a bounded linear mapping of $X$ into $X^{*}$ such that $f(x)=(A x, x)$ is weakly lower semicontinuous. If $N$ is a pseudo-monotone mapping of $X^{*}$ into $X$, then the mapping $T=A^{*}+A N A^{*}: X \rightarrow X^{*}$ is also pseudo-monotone.

Proof. Let $\left\{x_{n}\right\} \subset X$ be a sequence such that $x_{n} \rightarrow x_{0}$ in $X$ and

$$
\limsup _{n}\left(T x_{n}, x_{n}-x_{0}\right) \leqq 0 \text {. }
$$

Since $A^{*}$ is linear and bounded, $A^{*} x_{n} \rightarrow A^{*} x_{0}$ in $X^{*}$ and, therefore, $\left(A^{*} x_{0}, x_{0}\right)$ $\leqq \lim \inf _{n}\left(A^{*} x_{n}, x_{n}\right)$ by our assumption on $f(x)$. This and the equality

(+) $\left(N A^{*} x_{n}, A^{*} x_{n}-A^{*} x\right)=\left(A N A^{*} x_{n}, x_{n}-x\right)=\left(T x_{n}, x_{n}-x\right)-\left(A^{*} x_{n}, x_{n}-x\right)$

imply that, letting $x=x_{0}$ in $(+)$,

$$
\begin{aligned}
\lim \sup & \left(N A^{*} x_{n}, A^{*} x_{n}-A^{*} x_{0}\right) \\
& \leqq \lim \sup \left(T x_{n}, x_{n}-x_{0}\right)-\lim \inf \left\{\left(A^{*} x_{n}, x_{n}\right)-\left(A^{*} x_{n}, x_{0}\right)\right\} \leqq 0 .
\end{aligned}
$$

Since $N$ is pseudo-monotone we may conclude that for all $u$ in $X^{*}$ and, in particular, for all $u \in R\left(A^{*}\right)$,

$$
\left(N A^{*} x_{0}, A^{*} x_{0}-A^{*} x\right) \leqq \liminf _{n}\left(N A^{*} x_{n}, A^{*} x_{n}-A^{*} x\right), \quad x \in X
$$

from which, in view of the equality $(+)$, we obtain the inequality

$(++)\left(T x_{0}, x_{0}-x\right) \leqq \lim \inf \left(T x_{n}-A^{*} x_{n}, x_{n}-x\right)+\left(A^{*} x_{0}, x_{0}-x\right), \quad x \in X$.

Again, since $A^{*} x_{n} \rightarrow A^{*} x_{0}$ and $f(x)=\left(A^{*} x, x\right)$ is weakly lower semicontinuous, for any $x$ in $X$ we have

$\lim \inf \left\{\left(A^{*} x_{n}, x_{n}\right)-\left(A^{*} x_{n}, x\right)\right\} \geqq \liminf \left(A^{*} x_{n}, x_{n}\right)-\left(A^{*} x_{0}, x\right) \geqq\left(A^{*} x_{0}, x_{0}-x\right)$.

This and $(++)$ imply that for each $x$ in $X$

$$
\begin{aligned}
\left(T x_{0}, x_{0}-x\right) \leqq & \lim \inf \left(T x_{n}, x_{n}-x\right)-\lim \inf \left\{\left(A^{*} x_{n}, x_{n}\right)-\left(A^{*} x_{n}, x\right)\right\} \\
& +\left(A^{*} x_{0}, x_{0}-x\right) \\
\leqq & \lim \inf \left(T x_{n}, x_{n}-x\right) .
\end{aligned}
$$

It is easy to see that since $A^{*}$ is a bounded linear mapping of $X$ into $X^{*}$ and $N$ is a mapping of $X^{*}$ into $X$ which satisfies condition (ii) of Definition $3, T=A^{*}+A N A^{*}$ of $X$ into $X^{*}$ also satisfies condition (ii) of Definition 3. Hence $T=A^{*}+A N A^{*}$ is a pseudo-monotone mapping of $X$ into $X^{*}$. Q.E.D.

THEOREM 13. Let $X$ be a reflexive Banach space with a Schauder basis and with $X^{*}$ strictly convex. Let $A$ be a linear monotone mapping of $X$ into $X^{*}$ such that $\left\|A^{*} x\right\| \geqq \alpha\|x\|$ for all $x$ in $X$ and some $\alpha>0$. Let $N$ be a mapping of $X^{*}$ into $X$ such that for some constants $c \geqq 0, r>0$ and $0<l<1$,

$$
\begin{gathered}
(N u, u) \geqq(N(0), u)-c\|u\| \text { for all } u \in X^{*} \text { and some } c \geqq 0 \\
\|A N u\| \leqq l\|u\| \text { for all } u \text { in } X^{*} \text { such that }\|u\|>r
\end{gathered}
$$


Then the equation $u+A N u=f$ has a solution in $X^{*}$ for each $f$ in $X^{*}$ provided $N$ also satisfies any one of the following three conditions:

(A) $N$ is hemicontinuous and monotone.

(B) $N$ is bounded and pseudo-monotone.

(C) $N$ is bounded and of type (M) and $X$ has a weakly continuous duality mapping $J$ of $X$ into $X^{*}$.

Proof. We first note that since $A^{*}$ is a continuous monotone mapping of $X$ into $X^{*}$ such that $\left\|A^{*} x\right\| \geqq \alpha\|x\|$ for all $x$ in $X$, Proposition 5.1(a) implies that $A^{*}$ is onto and, of course, one-to-one. Thus, to show that $I+A N$ maps $X^{*}$ onto $X^{*}$, it suffices to show that $T=A^{*}+A N A^{*}$ maps $X$ onto $X^{*}$. This we now prove in each of the three cases.

(A) Since $A^{*}$ and $N$ are monotone, $T=A^{*}+A N A^{*}$ is also a monotone mapping of $X$ into $X^{*}$ which obviously is hemicontinuous. Moreover, (5.4) and the fact that $\left\|A^{*} x\right\| \geqq \alpha\|x\|$ for all $x$ in $X$ imply that for all $x$ in $X$

$$
\|T x\| \geqq\left\|A^{*} x\right\|-\left\|A N\left(A^{*} x\right)\right\| \geqq(1-l)\left\|A^{*} x\right\| \geqq(1-l) \alpha\|x\|
$$

from which we conclude that $\|T x\| \rightarrow \infty$ as $\|x\| \rightarrow \infty$. Thus, applying Proposition 5.1(a) and Remark 5.1 we find that $T$ maps $X$ onto $X^{*}$.

(B) Since $A$ is a linear monotone mapping of $X$ into $X^{*}$, the functional $q(x)$ $=\left(A^{*} x, x\right)$ is weakly lower semicontinuous. Hence, in view of our condition (B), Proposition 5.2 shows that $T=A^{*}+A N A^{*}$ is a bounded pseudo-monotone mapping of $X$ into $X^{*}$. Now, (5.3) and the monotonicity and boundedness of $A$ imply that for all $x$ in $X$

$$
(T x, x)-(T(0), x)=\left(A^{*} x, x\right)+\left(N A^{*} x, A^{*} x\right)-(N(0), x) \geqq-c^{\prime}\|x\|
$$

with $c^{\prime}=c\|A\| \geqq 0$. Since, by (5.4), $\|T x\| \rightarrow \infty$ as $\|x\| \rightarrow \infty$, Proposition 5.2(b) implies that $T$ maps $X$ onto $X^{*}$.

(C) In view of condition (C), Proposition 1.4 implies that $T=A^{*}+A N A^{*}$ is of type (M) and bounded which, by (5.3) and (5.4), is such that $(T x, x) \geqq(T(0), x)$ $-c^{\prime}\|x\|$ for all $x$ in $X$ and $\|T x\| \rightarrow \infty$ as $\|x\| \rightarrow \infty$. Since in (C) we also assume that $X$ has a weakly continuous duality mapping, Proposition 5.1(c) implies that $T$ maps $X$ onto $X^{*}$. Q.E.D.

In case $N$ is a bounded continuous and monotone mapping of $X^{*}$ into $X$, Theorem 13(A) has been established in [9].

\section{REFERENCES}

1. H. Amann, Über die Existenz und iterative Berechnung einer Lösung der Hammersteinschen Gleichung, Aequationes Math. 1 (1968), 242-266. MR 38 \#4933.

2. —_, Ein Existenz- und Eindeutigkeitssatz für die Hammersteinsche Gleichung in Banachräumen, Math. Z. 111 (1969), 175-190.

3. —_ Zum Galerkin-Verfahren für die Hammersteinsche Gleichung, Arch. Rational Mech. Anal. 35 (1969), 114-121.

4. F. E. Browder and C. P. Gupta, Monotone operators and nonlinear integral equations of Hammerstein type, Bull. Amer. Math. Soc. 75 (1969), 1347-1353. MR 40 \#3381. 
5. F. E. Browder, D. G. DeFigueiredo and C. P. Gupta, Maximal monotone operators and nonlinear integral equations of Hammerstein type (to appear).

6. H. Brezis, Equations et inéquations non-linéaires dans les espaces vectoriels en dualité, Ann. Inst. Fourier (Grenoble) 18 (1968), 115-176.

7. C. L. Dolph, Nonlinear integral equations of Hammerstein type, Trans. Amer. Math. Soc. 66 (1949), 289-307. MR 11, 367.

8. C. L. Dolph and G. J. Minty, On nonlinear integral equations of the Hammerstein type, Proc. Advanced Sem. Nonlinear Integral Equations (University of Wisconsin, 1963), Univ. of Wisconsin Press, Madison, Wis., 1964, pp. 99-154. MR 28 \#4322.

9. D. G. DeFigueiredo and C. P. Gupta, Solvability of nonlinear integral equations of Hammerstein type (to appear).

10. M. Golomb, On the theory of nonlinear integral equations, integral systems and general functional equations, Math. Z. 39 (1935), 45-75.

11. C. P. Gupta, On existence of solutions of nonlinear integral equations of Hammerstein type in Banach space (to appear).

12. A. Hammerstein, Nichtlineare Integralgleichungen nebst Anwendungen, Acta Math. 54 (1930), 117-176.

13. R. Inglisch, Existenz und Eindeutigkeit Sätze bei nichtlineare Integralgleichungen, Math Ann. 108 (1933), 161-189.

14. I. I. Kolodner, Equations of Hammerstein type in Hilbert spaces, J. Math. Mech. 13 (1964), 701-750. MR 30 \#1415.

15. J. Kolomy, Application of some existence theorems for the solutions of Hammerstein integral equations, Comment. Math. Univ. Carolinae 7 (1966), 461-478. MR 35 \#686.

16. - The solvability of nonlinear integral equations, Comment. Math. Univ. Carolinae 8 (1967), 273-289. MR 35 \#5878.

17. M. A. Krasnosel'skiī, Topological methods in the theory of nonlinear integral equations, GITTL, Moscow, 1956; English transl., Macmillan, New York, 1964. MR 20 \#3464; MR 28 \#2414.

18. V. V. Nemyckiî, Théorèmes d'éxistence et l'unicité des solutions de quelques équations intégrales non-linéaires, Mat. Sb. 41 (1934), 438-452.

19. W. V. Petryshyn, On existence theorems for nonlinear equations involving noncompact mappings, Proc. Nat. Acad. Sci. U.S.A. 67 (1970), 326-330.

20. L. B. Rall, Variational methods for nonlinear integral equations, Proc. Advanced Sem. Nonlinear Integral Equations (University of Wisconsin, 1963), Univ. of Wisconsin Press, Madison, Wis., 1964, pp. 155-189. MR 29 \#3912.

21. E. Rothe, Weak topology and nonlinear integral equations, Trans. Amer. Math. Soc. 66 1949), 75-92. MR 11, 184.

22. M. M. Vaĭnberg, Variational methods for the study of non-linear operators, GITTL, Moscow, 1956; English transl., Holden-Day, San Francisco, Calif., 1964. MR 19, 567; MR 31 \#638.

23. - New theorems for nonlinear operators and equations, Moskov. Oblast. Ped. Inst. Učen. Zap. 77 (1959), 131-143.

24. - Nonlinear equations with potential and monotonic operators, Dokl. Akad. Nauk SSSR 183 (1968), 747-749=Soviet Math. Dokl. 9 (1968), 1427-1430. MR 39 \#832.

25. M. M. Vaĭnberg and I. M. Lavrent'ev, Equations with monotonic and potential operators in Banach spaces, Dokl. Akad. Nauk SSSR 187 (1969), 711-714=Soviet Math. Dokl. 10 (1969), 907-910.

\section{RUTGERS UNIVERSITY,}

New Brunswick New Jersey 08903 